\title{
Questes
}

Revue pluridisciplinaire d'études médiévales

\section{Lettres de Dieu, lettres du Diable : correspondances entre Terre, Ciel et Enfer}

\section{Fanny Oudin}

\section{(2) OpenEdition}

1 Journals

\section{Édition électronique}

URL : http://journals.openedition.org/questes/1307

DOI : 10.4000/questes. 1307

ISSN : 2109-9472

\section{Éditeur}

Les Amis de Questes

\section{Édition imprimée}

Date de publication : 15 septembre 2010

Pagination : 37-55

ISSN : 2102-7188

\section{Référence électronique}

Fanny Oudin, «Lettres de Dieu, lettres du Diable : correspondances entre Terre, Ciel et Enfer », Questes [En ligne], 19 | 2010, mis en ligne le 01 janvier 2014, consulté le 17 septembre 2020. URL : http:// journals.openedition.org/questes/1307 ; DOI : https://doi.org/10.4000/questes.1307

\section{(c) Association des amis de «Questes »}




\section{Lettres de Dieu, lettres du Diable : correspondances entre Terre, Ciel et Enfer \\ Fanny OUDIN}

Parmi les différentes formes de manifestations divines, certaines, attestées dans la Bible et bénéficiant d'une tradition ininterrompue, jouissent d'une certaine légitimité : théophanies et miracles sont des formes connues et reconnues de manifestation du divin. Quant aux reliques, elles sont des traces et résidus matériels de manifestations divines antérieures, susceptibles de devenir à leur tour le support de miracles post mortem. Qu'elles soient reconnues ne signifie pas que leur existence ne prête pas à controverse, que ce soit dans le cadre d'une procédure juridique visant à prouver l'authenticité particulière d'une relique ou d'un miracle donnés, ou dans celui d'une réflexion ontologique plus générale sur la nature de ces manifestations de Dieu. Mais l'ouverture d'un espace de discussion, fût-il polémique, témoigne déjà d'une forme de reconnaissance que toutes les manifestations surnaturelles ne possèdent pas. La nature des apocryphes par exemple, ne saurait prêter à confusion: ce sont des faux. Pour la plupart, leur absence d'authenticité ne fait pas vraiment débat. Le statut des lettres tombées du Ciel par exemple, dont il sera question ici, est plutôt l'objet d'affirmations tranchées et catégoriques. En témoignent les remontrances de l'évêque Licinianus de Carthagène à son confrère Vincentius d'Ibiza, qui forment la première attestation de l'existence de la lettre du Christ tombée du Ciel :

Absit ergo a sanctitate tua hoc credere ut epistolae nunc nobis mittantur a Christo. Sufficiat enim quod est locutus in prophetis, per seipsum et per suos apostolos. Nam et his non litteras transmittebat e caelo, sed spiritu sancto eorum corda replebat. [...] Et forte quia ipsa epistola, sicut simulator scripsit, de caelo 
descendit super altare Christi in memoria S. Petri apostoli, scito diaboli esse figmentum. ${ }^{1}$

Cette apparition dans les archives sous la forme d'une dénégation n'est pas anodine : en dehors des imprécations de la lettre elle-même contre ceux qui ne voudront pas croire à son authenticité, les manuscrits ne nous ont conservé aucun plaidoyer en faveur de celle-ci. Par exemple, ni la lettre initiale de Vincentius d'Ibiza ni sa réponse à Licinianus ne nous ont été transmises. Et aujourd'hui l'existence de tels textes nous paraît incongrue : on peine à imaginer quelle a pu être la réception d'une lettre tombée du ciel pour transmettre aux fidèles la volonté et les commandements divins.

Il existe des formes assez variées de manifestations épistolaires de Dieu ou du diable. Par contraste, le Moyen Âge ne semble guère avoir imaginé que les autres formes de surnaturel se prêtent au jeu de la communication épistolaire $^{2}$. Si le problème de la valeur accordée à ces lettres se pose, c'est donc dans un contexte strictement chrétien, où la Vérité, attribut divin, s'oppose à la fois au mensonge, à l'illusion et aux apparences, instruments employés par le diable pour régner sur le monde terrestre soumis à un mouvement perpétuel qui brouille les signes. Ce cadre de référence commun homogénéise les différentes formes de manifestations épistolaires du surnaturel au Moyen Âge. Or dans un contexte aussi manichéen, la polarisation des rapports entre authenticité et imposture est dépourvue de toute ambivalence. L'intervention de puissances surnaturelles chrétiennes comme locuteurs suppose l'exclusion de la fiction et de ses ambiguïtés dans la problématique de la réception. Mais même si elles ne sont pas intrinsèquement fictives, ces lettres sont

\footnotetext{
${ }^{1}$ Cité d'après Hippolyte Delehaye, « Note sur la légende de la lettre du Christ tombée du ciel », in Mélanges d'hagiographie grecque et latine, Bruxelles, Subsidia Hagiographica $\mathrm{n}^{\circ} 42,1966$, p. 150-178.

2 À ma connaissance, ni les romans d'Antiquité ni les romans arthuriens ne mettent en scène de correspondance entre le monde humain et l'au-delà.
} 
souvent introduites par des éléments narratifs, voire insérées dans des cadres de natures variées, allant de la chronique au recueil de miracles. Leur existence a généré la création d'un motif, celui de la missive divine ou diabolique qui se retrouve dans des genres littéraires comme le roman, le théâtre ou le dit. Ces glissements invitent à se pencher de plus près sur la nature des textes en question.

La première lettre à circuler entre Ciel, Terre et Enfer est celle attribuée au Christ, qui serait tombée du Ciel pour recommander aux fidèles, avec force menaces et promesses, de respecter le repos du dimanche et, subsidiairement, de payer la dîme. Cet apocryphe, dont les premières mentions remontent $\mathrm{au} \mathrm{VI}^{\mathrm{e}} \operatorname{siècle}^{3}$, a joui d'une très large diffusion dans la chrétienté médiévale ${ }^{4}$, au point que les principaux débats qu'il a suscités portent sur son origine ${ }^{5}$. Sans se prononcer sur cette épineuse question, il est intéressant de considérer le point de chute que se donne la lettre lorsqu'elle met en scène sa propre réception. S'impose d'abord le caractère spectaculaire, et fortement symbolique de ces apparitions, souvent accompagnées de trois jours de jeûne et de prières qui font écho aux recommandations du texte. Voici par exemple le préambule du ms. BNF, lat. $12315^{6}$,

Epistola domini nostri Iesu Christi quae de caelis descendit super altare sancti Petri in Iherusalem, inscripta in tabulis marmoreis, et

\footnotetext{
${ }^{3}$ La plus ancienne est la condamnation de Licinianus de Carthagène. Elle réapparaît ensuite au Concile de Latran en 745, puis dans un capitulaire de Charlemagne en 789.

${ }^{4}$ Les différents articles qui lui sont consacrés mentionnent des textes latins, allemands, islandais, français, espagnols, occitans, italiens, grecs, éthiopiens, arabes, syriaques, slaves...

${ }^{5}$ Les tenants d'une origine orientale, avec un texte primitif rédigé en grec, s'opposent à ceux d'un modèle occidental, écrit en latin. Pour un aperçu de ces débats, voir l'article d'Hippolyte Delehaye déjà cité et le compte-rendu par Norman H. BAYNES de l'ouvrage de Robert PRIEBSCH, Letter from Heaven on the Observance of the Lord's Day, Oxford, 1936, paru dans Modern Language Review, 32 (1937), p. 649-651.

${ }^{6}$ Toutes les citations de la version latine sont tirées de l'article d'Hippolyte DELEHAYE.
} 
lumen de ipsa sicut fulgur erat. Angelus autem domini eam tenebat in manibus, et omnis populus cum videret eam, prae timore ceciderunt in facies suas, et clamantes dixerunt: Kyrie eleyson.

ou celui qui précède la lettre telle qu'elle est rapportée à Latran en 745,

In dei nomine incipit epistola domini nostri Iesu Christi filii Dei, que in Hierosolyma cedidit, et per Michael Arcangelum ipsa epistola inventa est ad portam Effrem. Et per manus sacerdotis nomine Icore epistola ista fuit relecta ipsa exemplata, et transmisit ipsam epistolam ad Geremiam civitatem ad alio sacerdoti Talasio. Et ipse Talasius transmisit ipsam epistolam ad Arabiam civitatem alio sacerdoti Leoban et ipse Leobanus transmisit epistolam ipsam ad Vetfaniam civitatem; et recepit epistolam istam Macrius sacerdos Dei et transmisit istam epistolam in monte sancto arcangelo Michael. Et ipsa epistola per manus angeli domini pervenit ad Romanam civitatem ad locum sepulcri sancti Petri ubi claves regni caelorum constitutae sunt. Et 12 papati qui sunt in Romana civitate, triduanas fecerunt vigilias in ieiuniis, in orationibus, per diebus et noctibus.

La première fonction de ces « récits-cadres », s'ils méritent un tel nom, est de donner un modèle d'efficacité performative aux commandements divins, en montrant les premiers lecteurs de la lettre en train d'exécuter à la fois les recommandations morales et les prescriptions plus spécifiques visant la diffusion de l'épître. Ces dernières sont le plus souvent liées à des protestations véhémentes de vérité, sous la forme de serments hyperboliques appelant à témoin toute la hiérarchie chrétienne, à la fois céleste et terrestre. Le statut de ce passage peut être ambigu, car il intervient à la toute fin de la lettre, et un prêtre y prend parfois la parole, comme dans le ms. Munich, lat. $9550^{7}$ :

Praecipio vobis sacerdotis meis ut unusquisque istam epistolam ostendat populo suo, et affirmate illis a me transmissam. Quod si non crediderint, anathematizabo eos usque in saeculum. Ego

\footnotetext{
${ }^{7}$ Ce serment se retrouve dans la version anglo-normande éditée par Clovis BRUNEL, « Versions espagnole, provençale et française de la lettre du Christ tombée du ciel », Analecta Bollandiana, t. 68 (1950), p. 383-386.
} 
Petrus episcopus indignus, iuro per Maiestatem Dei qui fecit caelum et terram, mare et omnia quae in eis sunt, per Ihesum Christum et per sanctam genetricem Mariam, per omnes angelos Dei, per omnes patriarchas, prophetas, apostolos, martyres, confessores, virgines, per reliquias omnium sanctorum atque electorum Dei, quia ista epistola non formata est manu hominis neque scripta, sed est scripta digito Dei et Domini nostri Ihesu Christi, et est transmissa de septimo caelo et de throno Dei in terram.

Il est alors difficile de savoir si le passage doit être rattaché à la lettre ellemême, ou lu comme le post-scriptum d'un copiste, ce qui serait paradoxal puisque c'est précisément à ce moment que la lettre affirme avoir été écrite de la main même de Dieu, et envoyée depuis le trône céleste. À la forme traditionnelle du serment, qui convoque les garants dans un ordre d'importance décroissant, se superpose une image du mandataire en majesté au milieu de ses sujets qui participe à la mise en scène de l'autorité de la lettre. De plus, on peut voir dans ce mouvement descendant une représentation du trajet de la lettre. La chaîne qui passe des êtres célestes aux élus humains puis aux simples prêtres crée une sorte de pont entre Ciel et Terre qui rend plus crédible la transmission de la lettre, en multipliant les intermédiaires ${ }^{8}$.

En ce qui concerne le trajet suivi par la lettre, le caractère symbolique des lieux où elle est déposée est évident. La lettre apparaît dans des villes saintes importantes, Jérusalem ou Rome. C'est toujours sur une tombe, un berceau ou près d'une porte qu'elle est déposée, c'est-à-dire en des lieux qui incarnent le passage, et plus particulièrement pour les deux premiers le passage entre le monde terrestre et l'au-delà. La transgression

\footnotetext{
${ }^{8}$ Ce n'est pas là la fonction première de cette liste, mais seulement un effet possible qui irait dans le même sens que le serment : de même que celui-ci confère une autorité à la parole qu'il garantit, de même la présence d'intermédiaires rend plus vraisemblable l'existence d'une communication entre Ciel et Terre, d'autant plus que nombre des figures citées, comme les anges, les prophètes ou les prêtres ont précisément pour fonction de favoriser une telle communication.
} 
que représente la lettre est ainsi indirectement mise en valeur: c'est l'établissement d'une communication directe entre des sphères ontologiques séparées qui est recherché. Cependant les copistes s'appesantissent aussi sur les tribulations terrestres de la lettre, avec un passage de main en main scandé par la reprise des mêmes expressions. De ces trajets, résultent deux dynamiques. La première est une translatio, d'Orient en Occident, qui mène de Jérusalem au Mont-Saint-Michel. Ce choix n'est pas anodin, à la fois parce que le Mont-Saint-Michel peut représenter une limite occidentale du monde des vivants et parce que le lieu est consacré à l'archange supposé avoir été le messager divin initial. Avec cette destination, se forme donc, au moins sur le plan onomastique, un cercle virtuel qui peut évoquer une sorte de perfection ou d'achèvement du parcours de la missive. Mais les récits mentionnent ensuite Rome ${ }^{9}$. Cette juxtaposition conjugue un imaginaire du transfert de pouvoir d'Orient en Occident avec une vision du monde qui sépare les deux sphères et les fait coexister, en décrivant l'arrivée simultanée de deux exemplaires de la même lettre, l'un en Orient, l'autre en Occident. Est mise en exergue la valeur universelle de la lettre, adressée à l'ensemble des croyants, et la nécessité de sa diffusion, là encore actualisée par le récit : 1'ordre formulé sur le mode impératif à la fin de la lettre est présenté par le récit initial comme déjà accompli. Enfin, reste à noter la participation de ces itinéraires au souci d'authentification. En effet, retracer pas à pas le parcours de la lettre en nommant chacun des intermédiaires les plus humbles contribue, en amassant des informations, à lui créer une identité. L'attention accordée aux noms propres n'est pas anodine. Elle fait partie intégrante de l'écriture épistolaire : par ce jeu de nominations, la lettre se donne un ancrage dans

\footnotetext{
${ }^{9}$ Pour Hippolyte Delehaye, du point de vue de la diffusion, cette conjonction de deux points de chute différents tient à l'amalgame fait très tôt entre deux versions de l'apocryphe.
} 
un contexte particulier qui la caractérise en propre comme forme littéraire $^{10}$. Entre autres, le nom propre a pour effet de créer une illusion référentielle. En ce sens, la litanie de noms propres, comme la liste des grades célestes, participe à l'authentification du texte.

Cette lettre céleste est assez différente de celle qui apparaît, à la même époque, dans la légende d'Abgar : au temps de l'incarnation Abgar, roi d'Edesse gravement malade, envoie une lettre au Christ car il désire fortement le voir. Le Christ lui répond qu'il lui enverra un de ses disciples après sa Passion, et lui fait parvenir en attendant un drap sur lequel figure son portrait en pied. Après la mort du Christ, l'un de ses disciples arrive effectivement à Edesse, où il guérit le roi et convertit le peuple. Dans la version occitane de la légende ${ }^{11}$, le récit s'achève sur les vertus miraculeuses de la lettre :

Pois que le reis ac receupuda la pistola de la man de nostre Seinnor Jesu Christ, nulla genz estrainna poc envazir la ciutat ni far nuil mal.

Cette ultime phrase constitue le principal point commun entre la lettre d'Abgar et celle tombée du Ciel, dont la version provençale éditée par Clovis Brunel se conclut sur une idée similaire :

Et qui creyra fermament que aquest loc hon aquesta pistolha sia ligida e publicada los dimenges en las gleyhas, los demonis jamays no veyran, e sy en aquel loc es lo demoni, encontenen s'in hira e jamay non i tornara, ni de mort sobitana no mora.

\footnotetext{
${ }^{10}$ De ce point de vue, il peut être utile de rapprocher la lettre du Christ du Respit de la Mort de JEAN LE FEVRE qui joue sur l'idée de correspondances entre Ciel et Terre de manière détournée et indirecte (cf. infra). L'édition de Geneviève HASENOHR montre bien comment, au fil du temps, la dimension personnelle et l'ancrage particulier du texte sont gommés précisément par l'effacement des noms propres. Or ceux-ci figurent tous au sein de la lettre ou dans des scènes qui ont trait à sa réception. Il semblerait donc que le nom propre fasse partie intégrante de la rhétorique épistolaire.

11 Camille Chabaneau et Georges Reynaud, «Légendes pieuses en provençal », Revue des Langues Romanes, t. 34 (1890), p. 227-230.
} 
Toutes deux ont donc été transformées en talismans, une utilisation dérivée prévue par le texte lui-même, peut-être parce qu'à l'époque où ces deux versions ont été transcrites, de telles utilisations existaient déjà. Cependant, l'utilisation magique de l'écrit n'est pas propre à ces lettres, et reste indépendante de leur contenu ${ }^{12}$. En revanche, ces correspondances relèvent de dynamiques distinctes avec d'un côté une communication nettement individualisée et initiée lors de l'incarnation terrestre du Christ, donc horizontale, immanente, et de l'autre, la transmission verticale, comme le dit l'appellation de lettre «tombée du Ciel», d'une lettre publique et collective. De plus, dans la légende d'Abgar, la lettre accompagne un objet merveilleux, et sert de paratexte à un miracle indépendant d'elle : c'est moins l'écrit qui se trouve investi de valeurs magiques, que l'image ${ }^{13}$. La lettre tombée du ciel au contraire est une missive dont l'existence même est un miracle : elle est événement autant que texte ou objet.

De ce point de vue, la lettre du Christ paraît plus proche d'un autre apocryphe, la lettre du diable au clergé : toutes deux sont des lettres adressées à un destinataire collectif qui dénoncent les péchés des hommes à travers la fiction d'une correspondance entre la Terre et l'au-delà, Ciel ou

\footnotetext{
${ }^{12}$ Voir Joseph-Claude Poulin, « Entre magie et religion. Recherche sur les utilisations marginales de l'écrit au Moyen Âge », in Pierre Boglioni (dir.), La Culture populaire au Moyen Âge, Montréal, L'Aurore, 1979, p. 121-143. L'article se présente comme une typologie d'utilisations magiques de l'écrit dont il relie l'émergence à un «illettrisme fonctionnel » tout en soulignant d'une part que ces pratiques ne touchent pas seulement l'écrit, mais s'inscrivent dans un contexte plus vaste, et d'autre part que des clercs y participent : elles ne peuvent donc être rattachées simplement à une opposition entre culture populaire et culture savante.

${ }^{13}$ L'association de la lettre et du portrait est fréquente au Moyen Âge. Dans le recueil hagiographique occitan édité par Camille Chabaneau et Georges Reynaud, la légende d'Abgar est immédiatement précédée d'un récit portant sur les miracles qui entourent une représentation de la Passion du Christ. Il est donc probable que ce sont plutôt les vertus miraculeuses de l'image que celles de l'écrit qui sont mises en exergue, et que la lettre ne joue que le rôle d'un simple accompagnement, même si l'auteur se donne la peine de retranscrire intégralement le texte des lettres d'Abgar et du Christ.
} 
Enfer $^{14}$. La principale différence tient au ton de l'épître, qui peut aisément se justifier par l'identité du locuteur: au sérieux de l'épître christique, véhémente dans ses imprécations, répond le rire de la satire diabolique, qui pervertit la rhétorique épidictique dans un éloge ironique des vices du clergé. La dimension plus polémique de la lettre se marque par une restriction du groupe des destinataires, mise en valeur par l'évolution des salutations. L'adresse collective des lettres du XII ${ }^{\mathrm{e}}$ siècle,

tetricis notis inscriptam, in quibus Sathanas et omne inferorum satellitum multitudo gratias omni ecclesiastico coetui de Tartaro emittebant ${ }^{15}$,

se transforme au XIII ${ }^{\mathrm{e}}$ siècle en dialogue au sommet entre les princes des ténèbres et ceux de l'Église,

Principes tenebrarum princibus ecclesiarum salutem. ${ }^{16}$

Enfin Pierre de Ceffons, au XIV ${ }^{\mathrm{e}}$ siècle, confère toute sa portée au terme principes par une énumération des titres des deux interlocuteurs qui parodie la pompe des épîtres officielles :

Lucifer, princeps tenebrarum, tristicia profundi regens Acherontis imperia, dux Herebi, rex Inferni rectorque Gehenne, universis sociis regni nostri, filiis superbie, precipue moderne ecclesie principibus, de qua noster adversarius Jhesus Christus per prophetam predixit «Odivi ecclesiam malignantium» salutem quam vobis optamus, et nostris obedire mandatis ac prout incepistis legibus parere Sathane ac nostri juris precepta iugiter observare.

Cette évolution met en valeur la cible principale de l'épître, l'exercice du pouvoir temporel, qui compromet l'accomplissement de la mission

\footnotetext{
${ }^{14}$ Sur la lettre du diable, voir Gianni ZIPPEL, « La lettera del Diavolo al clero, dal secolo XII alla Riforma », Bollettino dell'Istituto storico per il Medioevo, Archivio Muratoriano, 70 (1958), p. 125-179 : tous les extraits sont cités d'après cet article.

${ }^{15}$ Guilaume De Malmesbury, Gesta Regum Anglorum, cité d'après Gianni ZiPPel, ibid. La lettre du diable contenue dans sa chronique semble être la plus ancienne version connue.

${ }^{16}$ Cette salutation, citée d'après le texte d'Eudes de Chériton, est reprise, avec des variations lexicales qui conservent cependant la même idée, dans toutes les versions du XIII ${ }^{\mathrm{e}}$ siècle.
} 
spirituelle des prêtres en les exposant aux péchés et aux vices mondains. À la restriction du groupe des destinataires correspond une réduction de l'espace de diffusion, puisqu'il s'agit d'une lettre latine, donc exclusivement occidentale, et qui n'a à ma connaissance pas été traduite en langue vulgaire. Cette audience restreinte est liée à la valeur politique de la lettre $^{17}$, dont témoigne également son environnement textuel. Avant de passer dans la littérature morale, elle se trouve d'abord insérée dans des chroniques, qui restent l'un de ses cadres favoris tout au long du Moyen Âge ${ }^{18}$.

Il y a là une différence majeure entre la lettre du diable, qui n'est d'abord que l'un des motifs d'un exemplum complexe ${ }^{19}$ et conquiert progressivement son autonomie, et celle du Christ, qui circule d'abord de façon autonome, et n'est intégrée que tardivement dans des récits plus vastes $^{20}$. Le diable se révèle aussi un maître de rhétorique, si l'on compare le latin souvent élégant de ses lettres modelées sur les artes dictaminis, à celui généralement plus sommaire des lettres christiques. L'attention portée à la forme, tout comme l'intégration aux récits cadres de débats philosophiques sur les rapports entre l'existence terrestre et l'au-delà, portent la marque d'une origine savante qui favorise l'entrée en littérature des lettres surnaturelles. Celle-ci s'accompagne d'un brouillage des

\footnotetext{
${ }^{17}$ Pour une analyse détaillée du contexte politique de ces lettres et des utilisations qui en ont été faites jusqu'à la Réforme, voir l'article de Gianni ZIPPEL, « La lettera del Diavolo al clero, dal secolo XII alla Riforma », art. cit.

${ }^{18}$ Par exemple, Gianni Zippel signale que la version qu'en donne Vincent de Beauvais se trouve, de manière significative, dans le Speculum Historiale.

${ }^{19}$ Chez Guillaume de Malmesbury, la lettre s'insère dans l'histoire de deux clercs qui vivent dans le péché et débattent sur la survie de l'âme après la mort en opposant les théories de Platon et d'Épicure. Aussi, lorsque l'un d'eux décède, son ami le veille et l'âme damnée remonte des Enfers pour lui décrire son supplice. La lettre du diable est apportée, à la fin du récit, comme preuve supplémentaire de la véracité de ses dires. À la suite de cette aventure, le second clerc se convertit à une vie pieuse...

${ }^{20}$ Le seul exemple cité par Hippolyte Delehaye est son insertion dans la chronique de Roger de Hovedene, reprise ensuite dans d'autres chroniques anglaises.
} 
frontières entre authenticité et faux, caractéristique de l'action diabolique ${ }^{21}$. Par rapport aux lettres christiques, les missives diaboliques développent donc deux traits essentiels : d'une part leur valeur politique souligne que le modèle de ces lettres est celui des interactions humaines, et de l'autre le développement des récits-cadres favorise leur ouverture aux différentes formes de la fiction.

De fait, au XII ${ }^{\mathrm{e}}$ siècle, l'épître surnaturelle devient, indépendamment de tout contenu, un motif narratif, et fait son entrée dans la littérature en ancien français avec deux textes, le Roman d'Eracle de Gautier d'Arras ${ }^{22}$ et le Miracle de Théophile de Gautier de Coinci, qui sera ensuite adapté pour le théâtre par Rutebeuf ${ }^{23}$. L'importance du modèle des correspondances officielles humaines pour les lettres surnaturelles est particulièrement patente dans les deux Miracles de Théophile, celui de Gautier de Coinci et celui de Rutebeuf. En effet, au-delà des différences impliquées par le

\footnotetext{
${ }^{21}$ Gianni Zippel cite le chroniqueur Matteo Villoni, qui émet un doute sur l'authenticité de cette lettre et y voit un instrument dans des transactions diplomatiques. Même si le rôle de la lettre comme son origine demeurent ambigus, elle est présentée comme un faux, et à l'issue des négociations il se trouve que le parti le plus décrié emporte la victoire, sans qu'il soit possible de savoir si c'est là un effet de la lettre ou non.

${ }^{22}$ En ce qui concerne la lettre de Dieu qui confère à Eracle trois dons au début du roman de Gautier d'Arras, je me permets de renvoyer à l'article que je lui ai consacré pour la journée d'étude de Questes sur Intus et Foris, à paraître aux PUPS, sous la direction de Manuel Guay, Marie-Pascale Halary et Patrick Moran. Il me semble que cette lettre joue entre autres le rôle d'une lettre d'introduction qui investit Eracle de sa charge de prophète. Elle authentifie sa mission de messager et représentant de Dieu, tout comme les lettres portées par certains messagers et ambassadeurs ne font que les confirmer dans leurs fonctions.

${ }^{23}$ Gautier D’Arras, Eracle, Guy Raynaud de Lage (éd.), Paris, Champion, CFMA, 1976, v. 229-278 ; GAUTIER DE CoINCI, Les Miracles de Nostre Dame, Frédéric KoENIG (éd.), Genève, Droz, 1966 ; Rutebeuf, Le Miracle de Théophile, Jean Dufournet (éd.), Paris, GF, 1987. Théophile est un clerc très pieux et dévoué à la Vierge qui, dépossédé de sa charge par le nouvel évêque, rend hommage au diable et garantit son engagement par une lettre. Au bout d'un certain temps cependant, il se repent et va prier la Vierge. Celle-ci, en souvenir de son ancienne dévotion, consent à récupérer la lettre compromettante et la lui rapporte. Il la remet alors à l'évêque, pour que son cas puisse servir à instruire les autres chrétiens des ruses du diable.
} 
passage du récit à la scène, tous deux relient la rédaction de la lettre à la même exigence de la part du diable. Chez Gautier de Coinci, il précise aux vers 391-405 :

Et se couvient sanz nule aloigne

Que bone chartre encor m'en doigne.

Maint crestien m'ont deceü : [...]

Mes honors prennent et reçoivent

Et puis après si me deçoivent

Luez droit qu'a confessïon viennent. [...]

Ja crestien mais ne querrai

Se n'en ai lettres et sael.

et chez Rutebeuf, aux vers 250-254,

\section{De toi aie lettres pendanz}

\section{Bien dites et bien entendanz ;}

Quar maintes genz m'en ont sorpris,

Por ce que lor lettres n'en pris ;

Por ce les veuil avoir bien dites.

À la « bone chartre » de Gautier répondent les « lettres ... bien dites et bien entendanz » de Rutebeuf : l'adjectif «bon » comme l'adverbe «bien», souligné par la triple répétition et par le doublet «bien dites et bien entendanz », renvoient à une lettre en «bonne et due forme », à valeur contraignante. La « lettre » de Théophile est plutôt ce que nous appellerions un contrat, une charte pour reprendre le terme de l'ancien français. C'est d'ailleurs ce mot qui, sans surprise, revient le plus souvent pour désigner le document compromettant : il apparaît onze fois chez Gautier de Coinci, dont deux à la rime avec "chartre» au sens de prison, et six chez Rutebeuf, dont une redoublé par «brief». Les autres termes sont « escrit», qui revient à trois reprises chez Gautier de Coinci, et «lettres », qui apparaît une fois chez ce dernier et trois fois chez Rutebeuf. Reste à souligner l'importance accordée au sceau dans la présentation de la lettre. On en trouve trois occurrences chez Gautier de Coinci, aux vers 404, 418 et 423 , juste après la rédaction de la lettre. Cette triple mention à des intervalles très rapprochés sert à souligner la fermeté de l'engagement pris 
par le clerc, et la difficulté consécutive à le rompre. Cet effet est explicitement recherché par le diable, avec l'idée sous-jacente que l'écrit, ainsi dûment authentifié, rend impossible la rupture du contrat par la prière, au contraire de ce qui se produirait pour un simple engagement oral.

Chez Rutebeuf le sceau n'apparaît que deux fois, au vers 426,

Li Maufez en a lettres de mon anel empraintes, et au vers 652 ,

De l'anel de son doit seela ceste lettre,

De son sanc les escrist, autre enque n'i fist metre.

Mais ces mentions sont soutenues par l'attention prêtée aux signes qui, dans la présentation de la lettre, témoignent de sa valeur juridique et publique, comme dans l'expression «lettres pendanz ». Rutebeuf n'hésite pas à y ajouter des détails frappants comme l'emploi du sang en guise d'encre, qui souligne la force de l'engagement pris: la mutilation corporelle est révélatrice de l'enchaînement spirituel auquel se soumet Théophile. La différence d'accentuation entre Rutebeuf et Gautier de Coinci, qui insiste plutôt sur les valeurs sous-jacentes de l'« escrit», tient sans doute au changement de genre. Il est probable que lors de la représentation, le passage de la lettre de main en main, d'abord de Théophile au diable puis, sous la contrainte, du diable à la Vierge, et en sens inverse de la Vierge à Théophile, permet de rendre visible et palpable la trajectoire spirituelle de Théophile. Quant au passage final de l'épître, devenue «lettre commune» (v. 640) du diable «a toz cels qui [la] verront $»^{24}$, des mains de Théophile à celles de l'évêque qui en donne une lecture publique à la communauté des croyants - les spectateurs du

\footnotetext{
${ }^{24}$ Sur le sens de cette métamorphose, voir Denis LALANDE, «De la "chartre" de Théophile à la "lettre commune" de Satan. Le Miracle de Théophile de Rutebeuf", Romania, 108 (1987), p. 548-558. Il souligne qu'à la fin de la pièce Théophile, de protagoniste actif, devient l'enjeu et l'objet d'une lutte entre des puissances qui le dépassent.
} 
Miracle -, il me paraît illustrer très concrètement la dynamique même qui est celle de l'exemplum: faire d'une histoire singulière un avertissement profitable au plus grand nombre à travers sa mise en forme par une parole autorisée, celle du prêtre ${ }^{25}$. En ce sens, le brief $^{26}$ de Théophile a bien pour fonction de résumer l'ensemble de la pièce au moment d'en tirer la morale.

Cette alliance étroite entre la dimension narrative de la lettre et son rôle de publication se retrouve dans le plus ancien texte en ancien français qui insère une lettre, la Vie de saint Alexis. Seule l'épître rédigée par Alexis au moment de mourir permet à ses parents de reconnaître leur fils dans le mendiant qu'ils ont hébergé, alors qu'une voix divine a déjà rendu publique sa sainteté ${ }^{27}$. Cette valeur d'officialisation de la lettre est mise en relief par les circonstances de la lecture : seul le Pape a pu ouvrir la main du saint pour se saisir de la lettre, qu'il fait lire à haute voix par un chapelain, dont c'est le rôle officiel :

Li apostolie tent sa main a la cartre ;

Sainz Alexis la sue li alascet :

Lui le consent ki de Rome esteit pape.

Il ne la list ne il dedenz ne guardet:

Avant la tent ad un boen clerc e savie.

Li cancelers, cui li mesters en eret,

Cil list le cartre ; li altra l'esculterent. ${ }^{28}$

Il ressort de tous ces textes que la lettre, comme manifestation divine, présente une double caractéristique. D'une part, c'est une manifestation hybride, qui tient à la fois du miracle et de la relique.

\footnotetext{
${ }^{25}$ Puisqu'à l'origine les exempla réunis en recueils sont destinés à être intégrés aux sermons, donc à une lecture publique en chaire dans le cadre de la prédication.

${ }^{26}$ Rutebeuf est le seul à employer ce terme, qui n'apparaît pas chez Gautier de Coinci, tout comme il est le seul à transcrire le texte de la lettre lue à haute voix, alors que Gautier de Coinci met plutôt l'accent sur les prières prononcées par le prêtre.

${ }^{27}$ En ce sens, cette lettre est bien liée à une manifestation divine, même si elle n'en est pas une en elle-même puisque, étant écrite par un humain, son existence n'a rien de miraculeux.

${ }^{28}$ La Vie de saint Alexis, Texte du Manuscrit de Hildesheim (L), Christopher STOREY (éd.), Genève-Paris, Droz, TLF, 1968, v. 371-377.
} 
Miracle, elle l'est par son mode d'apparition, parce qu'elle se présente d'abord comme un événement avant d'être un texte. Mais d'après l'importance accordée à l'objet, dont témoignent tant les textes que les utilisations dévoyées des apocryphes, la lettre divine peut aussi être traitée comme une relique. D'autre part, ces manifestations épistolaires sont visiblement pensées sur le modèle des correspondances officielles humaines, comme le montre la valeur contraignante accordée à la lettre de Théophile. Certes, dans ce miracle, l'origine de la lettre est humaine, et non surnaturelle, ce qui pourrait être considéré comme un facteur d'explication. Toutefois, à l'instar des lettres dont les mandataires sont des êtres infernaux ou divins, celle de Théophile n'engage pas des relations horizontales entre créatures terrestres, mais bien une relation verticale avec les puissances « célestes ».

On pourrait alors se demander si ce modèle des «lettres pendanz » ne serait pas un modèle exclusivement réservé aux correspondances diaboliques, tandis que les lettres divines iraient plutôt chercher le leur du côté de la pastorale. Cette hypothèse pourrait être soutenue par l'opposition faite par les textes eux-mêmes entre l'efficacité de la prière et celle de la chartre. Elle est très présente, avec un effet édifiant, chez Gautier de Coinci à travers le choix significatif de ne pas retranscrire la lettre alors que la prière consécutive de l'évêque est extrêmement développée. Chez Rutebeuf, elle est plus discrète et rappelle plutôt la visée satirique de l'épître diabolique en latin, car son effet est comique : le document censé garantir le diable contre tout repentir et attester sa puissance est lu pour démontrer son échec. L'insistance initiale du diable sur la garantie offerte par l'engagement écrit, qui servait d'abord à dramatiser le geste de Théophile, devient rétrospectivement amusante. Cette différence d'efficacité par rapport à la prière pourrait renvoyer au fait que la lettre, document typiquement humain, appartenant à l'univers terrestre donc 
soumis au changement, n'est douée que d'une pérennité relative, tandis que la prière est un discours sanctifié par son emploi exclusif dans le dialogue avec Dieu. Il ne faudrait pas, toutefois, en conclure à une opposition tranchée entre ces deux modes de communication, la parole vive et l'écrit à valeur juridique. Au contraire, l'opposition ponctuelle entre le contrat diabolique et la prière prend tout son sens sur fond d'un entremêlement des deux pratiques. Ainsi, dans Eracle, le «brief» divin qui tombe sur le berceau du nouveau-né s'inscrit dans un cycle de prières adressées par les parents à Dieu (v. 135-141). De même, dans les deux Miracles de Théophile, la restitution de la lettre se trouve encadrée par les prières, tout comme dans la découverte du corps et de la lettre dans la Vie de saint Alexis.

L'interaction et la complémentarité entre les prières et les lettres sont d'ailleurs proposées par Alain Boureau comme facteur d'explication de l'apparition de ces étranges apocryphes que sont les lettres divines ${ }^{29}$. De ce point de vue, les lettres du diable, dont l'apparition est plus tardive que

29 Alain BOUREAU, « La norme épistolaire, une invention médiévale », in Roger Chartier (dir.), La Correspondance. Les usages de la lettre au XIX $X^{e}$ siècle, Paris, Fayard, 1991, p. 127-157. Pour notre sujet, voir en particulier les p. 129-136. Selon lui, le modèle de la communication épistolaire avec le divin est fourni par les épîtres pauliniennes, dont l'importance dans la liturgie « tient à ce mélange de familiarité (Paul écrit à ses amis) et de sacralité (Paul transmet la parole de Dieu) [qui] représente bien l'originalité essentielle du Christianisme: l'Incarnation a fait venir Dieu sur terre ». Ainsi, tant les modalités de la lecture des épîtres pendant la messe, qui affirment le " présent de la communication » avec une « familiarité simple » et placent la lettre «à la jonction du divin et de l'humain », que la pratique des correspondances ecclésiastiques, inspirées par le modèle paulinien, favorisent l'idée qu'à travers les lettres d'hommes inspirés par le Saint-Esprit, c'est Dieu lui-même qui s'adresse aux fidèles. De la sorte en effet, «la possibilité d'une communication épistolaire avec le Ciel n'avait rien d'impensable » car « la lettre, le sermon, la prière, le droit pontifical procèdent d'un même ensemble persuasif, d'une norme générale de la Parole de l'Église » où le modèle de l'Épître, très productif, occupe une place de choix : «Paul a appris à l'Occident à croire (ou à devoir croire) en la puissance de la lettre qui vivifie ». De fait, les trois dons d'Eracle lui sont transmis par la lecture de la lettre. Pour un commentaire un peu plus approfondi de cette efficacité particulière du Verbe divin dans la lettre d'Eracle, voir l'article déjà cité à paraître dans Intus et Foris. 
celle des lettres du Christ, marquent un double glissement. D'une part la pratique épistolaire elle-même évolue vers une forme de plus en plus rhétorique et codifiée, avec l'apparition des artes dictaminis autour du $\mathrm{X}^{\mathrm{e}}$ siècle et leur développement aux $\mathrm{XII}^{\mathrm{e}}$ et $\mathrm{XIII}^{\mathrm{e}}$ siècles, qui coïncide avec l'apparition du corpus diabolique. D'autre part, peut-être en conséquence de cette évolution, le type épistolaire qui semble dominer de plus en plus n'est plus tant celui du sermon ${ }^{30}$ que celui de la juridiction pontificale ${ }^{31}$. Dans l'utilisation de la lettre comme motif narratif ou théâtral, le modèle juridique, tiré de l'exercice du pouvoir temporel de l'Église, prévaut, sans que cela paraisse incompatible avec l'insertion des lettres dans des cycles de prières. Il y aurait là tout simplement fusion de deux modèles, la lettre de prédication et la lettre pontificale, suivie d'une évolution vers une prédominance de la seconde quant aux valeurs attachées à l'emploi de la forme épistolaire. Cette confusion entre épîtres temporelles et spirituelles est portée à son comble par la fiction juridique originale inventée en 1376 par Jean le Fèvre dans le Respit de la Mort ${ }^{32}$. L'auteur, malade, imagine d'envoyer une lettre à ses collègues et amis du Parlement de Paris pour leur demander une lettre de répit contre Dieu, qui est son débiteur, et reculer ainsi l'heure de sa mort. Le poème s'achève de façon ludique en discréditant les répits royaux, qui ne peuvent être renouvelés, contrairement à la grâce octroyée par Dieu, souverain juge. Cette boutade finale témoigne du caractère de plus en plus littéraire des épîtres divines, qui finissent par n'être plus présentées que comme un jeu.

\footnotetext{
${ }^{30}$ Qui devient autonome avec le développement du sermon comme genre codifié disposant de ses propres manuels.

31 Qui se fait toujours sous forme de lettres, qu'il s'agisse de décrétales, de responsiones, ou d'encycliques.

32 Jean Le Fevre, Le Respit de la Mort, Geneviève Hasenohr (éd.), Paris, Picard, 1967.
} 
Cette forme fictive et ludique d'épîtres surnaturelles est celle qui se perpétuera ensuite avec des correspondances non plus entre la masse des fidèles et les puissances chrétiennes, mais entre les puissants de ce monde, patrons des poètes, et les illustres habitants des Enfers païens dont ils cherchent à égaler la gloire. L'œuvre de Jean Lemaire est particulièrement représentative de ce glissement vers le pur jeu littéraire, avec les célèbres Épîtres de l'Amant Vert adressées à Marguerite d'Autriche, depuis les Champs Élysées, par son perroquet décédé, ou la fiction d'une correspondance entre Hector et Louis XII inventée avec Jean d'Auton ${ }^{33}$. Ce changement de cadre de référence, avec le remplacement des sermons chrétiens sur le Salut par les figures souriantes de la mythologie antique, marque l'entrée définitive du motif des lettres venues de l'au-delà dans l'univers poétique, et leur fait quitter celui, plus ambigu, des apocryphes. Le corpus particulier des lettres surnaturelles présente donc une rupture très nette entre les écrits du Moyen Âge et ceux de la Renaissance. L'insertion de l'épître surnaturelle dans le cycle liturgique des prières est complètement abandonnée au profit d'un pur jeu littéraire qui renonce à toute évaluation en termes d'authenticité. Ce changement de thème et de statut fait ressortir, par contraste, l'homogénéité de l'ensemble formé par les lettres de Dieu et du diable, propres au Moyen Âge. Aucun lien ne paraît exister entre les correspondances surnaturelles du Moyen Âge et celles de la Renaissance, qui relèvent de deux démarches et même de deux genres différents. Toutefois, ce passage dans l'univers de la fiction et de la littérature semble déjà en germe dans l'évolution qui mène, à travers les épîtres diaboliques, de la lettre du Christ, véritable apocryphe, au Respit de

\footnotetext{
${ }^{33}$ Jean d'Auton est le Rhétoriqueur qui précède Jean Lemaire au poste d'historiographe d'Anne de Bretagne. Il a écrit une lettre d'Hector à Louis XII. Jean Lemaire rédige la réponse du roi à son illustre correspondant. Ces jeux s'inscrivent donc explicitement dans une dynamique de glorification qui cherche à égaler le souverain aux héros de l'Antiquité.
} 
la Mort, jeu de clerc qui ne prétend à aucun sérieux. Le développement du récit cadre semble jouer, dans cette entrée progressive en littérature, un rôle non négligeable. Il est toutefois logique que, par la suite, une cohérence ait été rétablie par la séparation entre d'une part la lettre apocryphe du Christ, qui s'est perpétuée jusqu'à l'époque contemporaine sous la forme d'un simple talisman, et d'autre part le type littéraire de la correspondance avec l'au-delà, dont l'autonomie est garantie par l'emploi de figures mythologiques, le sérieux des vérités chrétiennes s'accommodant mal des jeux ambigus de la fiction. 